\title{
Synthesis of Biodegradable Plastics from Dyeing Wastewater and Optimization of Continuous Poly(3-Hydroxybutyrate) Fibrous Membranes via Electrospinning Process
}

\author{
Yaohui Liu' ${ }^{*}$, Yanming Wang1 ${ }^{1}$ Cheng-Hao Lee ${ }^{2}$, Chi-Wai Kan², Hong Chua ${ }^{3}$ \\ ${ }^{1}$ Department of Construction Technology and Engineering, The Technological and Higher Educational Institute of Hong Kong, \\ Hong Kong, China \\ ${ }^{2}$ Institute of Textiles and Clothing, The Hong Kong Polytechnic University, Hong Kong, China \\ ${ }^{3}$ Chaoshan Research Institute, Chaozhou, China \\ Email: *yhliu@vtc.edu.hk
}

How to cite this paper: Liu, Y.H., Wang, Y.M., Lee, C.-H., Kan, C.-W. and Chua, H. (2020) Synthesis of Biodegradable Plastics from Dyeing Wastewater and Optimization of Continuous Poly(3-Hydroxybutyrate) Fibrous Membranes via Electrospinning Process. Journal of Materials Science and Chemical Engineering, 8, 71-79.

https://doi.org/10.4236/msce.2020.812006

Received: December 8, 2020

Accepted: December 24, 2020

Published: December 31, 2020

\begin{abstract}
The Polyhydroxyalkanoates (PHAs) are promising biodegradable materials exhibit similar thermal and mechanical properties as conventional plastics. However, the current production cost for PHAs is high and less application examples were found. In addition, the development of electrospun nanofibrous membrane has attracted more attention in recent years. This study aims to investigate the production of PHAs using dyeing wastewater as the carbon source to reduce the major operation cost and prepare Poly(3-hydroxybutyrate) (PHB)-based fibrous membrane with controlled surface microstructure through electrospinning technique. At the optimal Carbon to Nitrogen (C:N) ratio (100:1) by using dyeing wastewater, the reactor achieved $0.2562 \mathrm{~g}$ PHAs from unit mass of activated sludge and $0.0571 \mathrm{~g}$ PHAs from unit mass of COD consumed. By using industrial glucose as carbon source adjustment, $1.180 \mathrm{~g}$ polymer per $1 \mathrm{RMB}$ could be synthesized at the optimal C:N ratio. As a feasible study, the industrial PHB was used for electropining. The influence of the process parameters (applied electric field, solution flow rate and polymer concentration) on the electrospun fiber microstructure was investigated and optimized in order to achieve smooth, bead-free fibers with diameter range of $1-10 \mathrm{~mm}$. The morphological transition from bead-like microstructure to smooth fiber was investigated under control of three major process parameters: applied voltage increased from $9 \mathrm{kV}$ to $20 \mathrm{kV}$, flow rate decreased from $5 \mathrm{~mL} \cdot \mathrm{hr}^{-1}$ to $0.5 \mathrm{~mL} \cdot \mathrm{hr}^{-1}$ and polymer concentration increased from $9 \mathrm{wt} \%$ to $15 \mathrm{wt} \%$. It was observed that well-defined fibrous film was achieved at applied electric field of $12 \mathrm{kV}$, flow rate of $1 \mathrm{~mL} \cdot \mathrm{hr}^{-1}$ and solution
\end{abstract}


concentration of $14 \mathrm{wt} \%$. The optimally processed electrospun fibers exhibited large area, continuous network with good distribution of fiber diameter distribution. Fibrous membrane fabricated by well-developed electrospinning technique has potentially offered application interest in the design of barrier coatings, adhesive interlayers for biological materials, and membranes for filtration of particulate matters and fiber-based food packaging materials.

\section{Keywords}

Poly(3-Hydroxybutyrate), Electrospinning, Fibrous Membrane, Biodegradable

\section{Introduction}

Polyhydroxybutyrate (PHB) is a biodegradable, biocompatible, and non-toxic biopolymer produced by microbes from soluble substrates and consumed by bacteria as energy storage source under the conditions of excess amount of carbon and nutrient-deficient environment [1] [2] [3]. The synthesis of PHB is achieved with a high carbon to nutrient ratio. After complete utilization of nitrogen and phosphorus source, bacterial growth is inhibited and the energy source storage will participate in the synthesis of the PHB-based macromolecules.

The biodegradability and nontoxic nature of PHB become more attractive in various medical areas owing to its therapeutic properties [4]. Although the use of PHB has been well established in various industrial fields, its commercial success is still restricted by the high production cost [2].

Electrospinning is currently the promising technique in the fabrication of continuous fibrous membrane down to nanoscale [5]. Electrospinning generally affords well-defined fibers with smooth surface. Nanofibers with morphologies such as bead-on-string [6] [7], porous bead [8] [9] [10] [11], and multiple necking structures [12] are of great interest because of their intriguing properties, which are not able to be achieved with fibers in macroscopic scale. Essentially, the morphological transition of fibrous microstructures is getting considerable attention in the application of medical and environmental areas.

It is important to pay attention that the diameter and surface morphology of the polymeric fibers obtained by electrospinning approach is controlled by electrospinning parameters [13] [14]. In fact, there are several critical parameters relevant to the solution properties, such as the polymer concentration, electrical conductivity, solution viscosity, surface tension of the solution which can be optimized within a range of reasonable values for improving the electrospinning process [15]. Some electrospinning process parameters can be effectively tuned to alter the diameter and morphology of the fibers, for example, applied voltage, solution flow rate, tip nozzle-to-collector distance, humidity, and working temperature. In general, the polymer concentration is inversely proportional to the 
diameter of the electrospun fiber [16] [17]. Process parameters such as applied voltage and solution flow rate significantly alter the fiber morphology rather than the fiber diameter [18], while the polymer concentration is highly connected to the surface tension and viscosity [19] and correlated to the fiber diameter control. Combining the above considerations, high surface tension and low polymer solution viscosity facilitate the transition from smooth fiber to beaded fibers with nanoporous structures [19] [20] [21].

The electrospinning of fibrous membrane is a simple and comprehensive process wherein the fiber morphology and size in terms of diameter can be manipulated under different process parameters. The relationship between these parameters and corresponding fiber networks is yet to be addressed to obtain high quality smooth and ultrafine fibrous structures without defects.

\section{Experimental}

\section{PHAs Accumulation}

The original dyeing wastewater treatment process of an industrial park in Guangzhou, China, treats the raw sewage from dyeing industries through screening, preliminary sedimentation, anaerobic fluidized bed, oxidation ditch, secondary sedimentation and disinfection. The return activated sludge (RAS) of the treatment plant was taken as seeding sludge and the effluent from its anaerobic reactor effluent (348 $\pm 25 \mathrm{mg}$ COD/L) was served as carbon source for PHAs accumulation in a pilot scale Sequencing Batch Reactor (SBR) with an effective volume of $1.403 \mathrm{~m}^{3}$. After the system operation was stable to produce a treatment performance up to $90 \%$ removal of COD at C:N ratio of 20:1. The C:N ratio of influent was adjusted by external dosing of industrial glucose. The C:N ratio ranged from 20:1, 40:1, 60:1, 80:1, 100:1 to 120:1. The system was operated for 7 days ( 3 cycles a day). The settled sludge in a stable performance was taken for PHAs analysis by VARIAN 3380 Gas Chromatography equipped with a programmable autosampler VARIAN CP-8400 after pretreatment. The operational parameter monitoring including COD, $\mathrm{pH}$, MLSS, MLVSS, TOC and TKN was done for each cycle according to the APHA standard methods.

\section{Electrospinning}

To study the feasibility of application of PHAs in electrospinning technology. Poly(3-hydroxybutyrate) PHB, MW = 1,000,000 (Di Jie Plastics Co. Ltd., Suzhou, Jiangsu, China) was prepared into well-dispersed solution with the concentration $7-15 \mathrm{wt} \%$ via dissolution in chloroform. Before electrospinning process, standard $10 \mathrm{~mL}$ glass syringe with metal luer lock tip (SAMCO ${ }^{\mathrm{TM}}$ Co. Ltd., UK) was filled with PHB solution and continuously injected by syringe pump with the flow rate at $0.5,1,3$, and $5 \mathrm{~mL} / \mathrm{hr}$, respectively. To further investigate the effect of applied voltage, solution concentration and solution flow rate, the distance between the needle tip and rotating drum collector was fixed at $16 \mathrm{~cm}$. The polymer solution was driven out of the syringe using an NE-300 single syringe pump (Model TL-F6, Tong Li Tech. Co. Ltd. Shenzhen, China). A metal 23-gauge 
syringe needle (SAMCO ${ }^{\mathrm{TM}} \mathrm{Co}$. Ltd., UK) was attached to the syringe. A horizontal orientated experimental design was implemented with the rotational drum collector positioned towards the needle tip. The needle tip and drum collector were both connected to a high voltage power supply (Model TL-Pro, Tong Li Tech Co. Ltd., Shenzhen, China) with DC voltage setting up to $30 \mathrm{kV}$. The applied voltage was fixed at $12 \mathrm{kV}$ in the effect studies of flow rate and $\mathrm{PHB}$ concentration. A flow rate of $1 \mathrm{~mL} \cdot \mathrm{hr}^{-1}$ was optimized in order to create a stable spinner jet flow in which the force of solution accumulated into the drop equivalent to the mean rate of fluid ejection by the jet.

\section{Characterization}

Electrospun fibers were deposited with a thin gold layer using a metal sputtering coater (E-1010, Hitachi Co. Japan) and the morphological change was investigated by scanning electron microscopy (SEM) (TM 3000 Table Top SEM, Hitachi Co. Japan) with an accelerating voltage of $15 \mathrm{kV}$. The diameter range of the fibers was measured approximately 100 fibers using SEM image $(500 \times$ magnification) and the Image J software.

\section{Results and Discussion}

\section{PHAs Accumulation}

As shown in Figure 1, the $Y_{X / S}(\mathrm{~g} \mathrm{CDW} / \mathrm{g} \mathrm{COD}$ consumed) is the specific growth yield. The $Y_{P / X}$ (g polymer/g CDW) is the specific polymer yield. The $Y_{P / S}$ (g polymer/g COD consumed) is the overall polymer production. The relation among three of them is shown below.

$$
Y_{P / S}=Y_{X / S} \bullet Y_{P / S}
$$

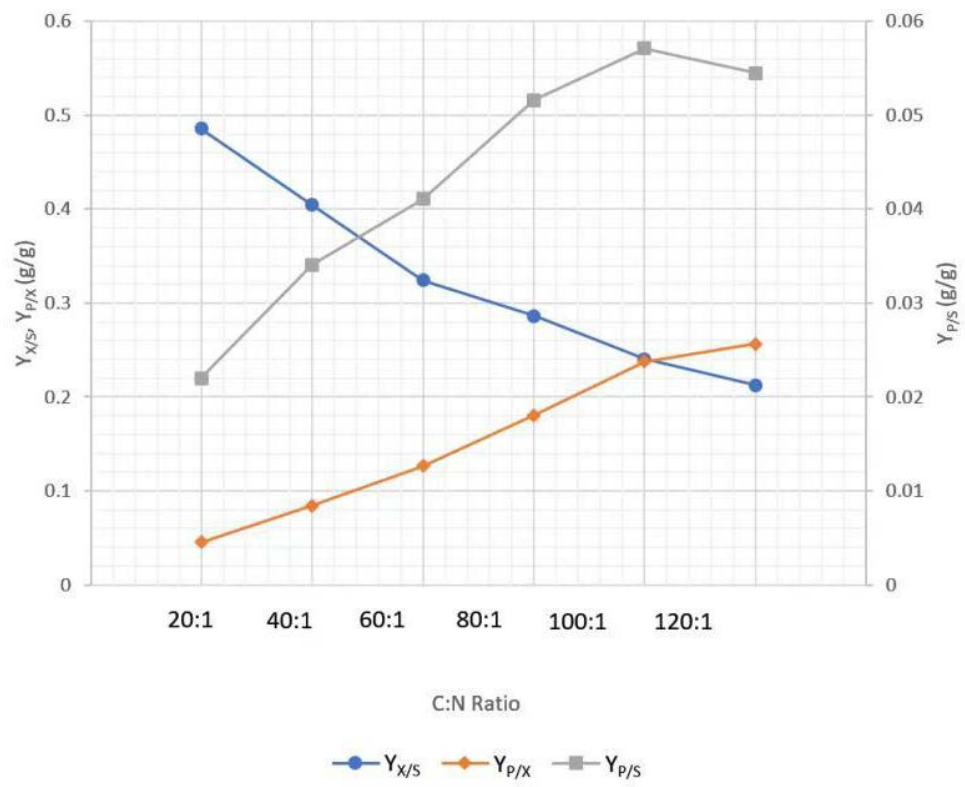

Figure 1. Specific growth yield ( $Y_{X / \Omega}, \mathrm{g}$ CDW/g COD consumed), specific polymer yield ( $Y_{P \mid X}, \mathrm{~g}$ polymer/g CDW) and overall polymer production $\left(Y_{P / S}\right.$ g polymer/g COD consumed) under various $\mathrm{C}: \mathrm{N}$ ratios. 
When the C:N ratio increases from 20:1 to $120: 1$, the specific polymer yield increases from 0.0294 to $0.2562 \mathrm{~g} / \mathrm{g}$. However, the specific growth yield decreases from 0.5252 to $0.2128 \mathrm{~g} / \mathrm{g}$. If considering the productivity of PHAs of unit COD consumed, the C:N ratio 100:1 will be the optimal point. The overall polymer production achieves $0.0571 \mathrm{~g} / \mathrm{g}$ at such C:N ratio.

The increasing C:N ratio forms the inhibition for the cell growth, so the PHAs accumulation was enhanced. As the C:N ratio increases from 20:1 to 120:1, the PHAs accumulated in specific cell were increased nearly linearly. High C:N resulted in high accumulation of PHAs in cells. However, the increasing C:N ratio also inhibited the growth of the cells. As a result, the specific growth yield decreased. When the C:N ratio was adjusted to be 100:1, the cells quantities and the PHAs amount in each cell reached an optimal level. If C:N ratio was increased higher than 100:1, the specific PHAs yield in each cell body also increased. However, the specific growth yield greatly decreased. As a result, the overall yield turned down.

In the pilot-scale study, the industrial glucose substrate was used for C:N ration adjustment. Under the optimal yield with C:N 100:1. If the dyeing wastewater was free for charge. The dosage cost production was estimated to be $1.180 \mathrm{~g}$ polymer per $1 \mathrm{RMB}$.

\section{PHB Electrospining}

\section{Applied Voltage}

The PHB nanofibers were spun in various applied voltages (ranged from $9 \mathrm{kV}$ to $20 \mathrm{kV}$ ) with other parameters were fixed (flow rate $1 \mathrm{~mL} \cdot \mathrm{hr}^{-1}$, concentration $14 \mathrm{wt} \%$ concentration, collect distance $16 \mathrm{~cm}$, temperature $25^{\circ} \mathrm{C}$ ). For $14 \mathrm{wt} \%$ PHB solution at a flow rate of $1 \mathrm{~mL} \cdot \mathrm{hr}^{-1}$, a voltage at $12 \mathrm{kV}$ leads to fibers with diameter ranged from 2 to $5 \mathrm{~mm}$ (Figure 2(a)). The fiber diameter distribution became narrow with the increasing applied voltage. The bead-on-string structure was almost vanished when the applied voltage was $12 \mathrm{kV}$. However, the fiber microstructure was not changed significantly even the voltage increased up to $20 \mathrm{kV}$. As a result, increasing applied voltages tend to inhibit the formation of fibers with bead-on-string morphology.

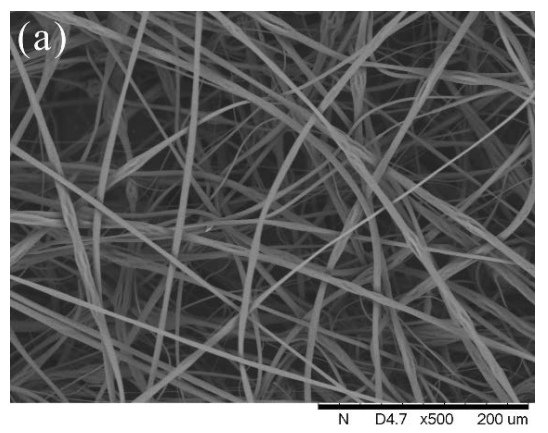

(b)

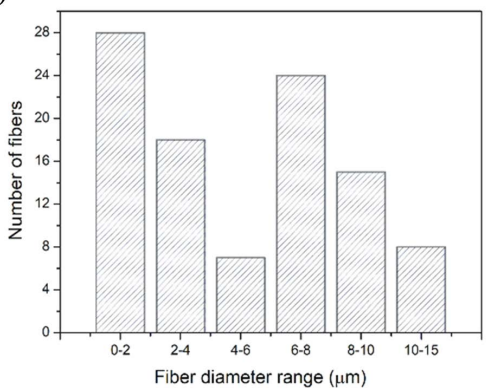

Figure 2. (a) SEM micrograph of electrospun poly(3-hydroxybutyrate) fibrous membrane obtained under applied voltage of $12 \mathrm{kV}$, solution flow rate of $1 \mathrm{~mL} \cdot \mathrm{hr}^{-1}$, solution concentration of $14 \mathrm{wt} \%$; (b) A histogram of average fiber diameter distribution. 


\section{Flow Rate}

The structural change of electrospun fibers was investigated by the effect of flow rate controlled by the syringe pump. The solution flow rate was varied from 0.5 to $5 \mathrm{~mL} \cdot \mathrm{hr}^{-1}$ (solution concentration $14 \mathrm{wt} \%$, applied voltage $12 \mathrm{kV}$, collect distance $16 \mathrm{~cm}$, process temperature $25^{\circ} \mathrm{C}$ ), respectively. The fiber diameter in the bead-on-string structure increased with increasing flow rate higher than 3 $\mathrm{mL} \cdot \mathrm{hr}^{-1}$. However, a continuous smooth fiber structure was observed at a flow rate at or below $1 \mathrm{~mL} \cdot \mathrm{hr}^{-1}$. As the flow rate was increased, the fiber morphology changed from smooth fiber into bead-on-string with marked change in the bead size. The findings indicated that the optimum flow rate of the PHB solution for well-defined continuous fiber should be maintained at $1 \mathrm{~mL} \cdot \mathrm{hr}^{-1}$ or below.

The results revealed that increasing solution flow rate decreases the mean fiber diameter. However, the fiber diameter distribution does not change obviously for the electrospun fiber samples collected under different flow rates. As the flow rate increases due to the larger volume of solution that is ejected from the nozzle in short period of time, bead diameter is expected to be increased. This increase of volume carried away from the nozzle tip will generate superfluous solution with high surface tension and the moving jet is not able to stretch completely. Consequently, beads distributed unevenly along the fiber were formed due to high surface tension and axisymmetric jet instabilities [22].

\section{Solution concentration in weight percentage}

The PHB solution concentration was varied from 9 to $15 \mathrm{wt} \%$ with other process parameters were kept constant (flow rate $1 \mathrm{~mL} / \mathrm{h}$, applied voltage $12 \mathrm{kV}$, nozzle-to-collector distance $16 \mathrm{~cm}$, temperature $25^{\circ} \mathrm{C}$ ).

The analysis reveals that the average fiber diameter value decreases correspondingly with a decrease in the polymer concentration. As the polymer solution concentration is very low, the stretched filaments between the droplets are stabilized and results in the formation of highly stable beads-on-string microstructure. The resultant morphology can be attributed to the transformation of the soluble polymer or macromolecules from elongated fibers due to spinner jet into entangled fiber networks under solvent evaporation and subsequent solidification processes. The jet radius was contracted owing to the surface tension as the driving force, leading to the formation of bead-like structures from the remaining solution.

The surface tension decreased correspondingly when the solution concentration is increased. Reduction of surface tension increases the surface area per unit mass of fiber, which favors jet thinning process and against the formation of bead-on-string structures. Meanwhile, the viscoelastic forces due to high solution concentration would be strong enough to compete with the Coulombic repulsion from the jet elongational flow and the charge interaction along the electric field. Hence, the resultant fiber diameter was significantly reduced [23].

It could be found that the bead structure was flattened and the fiber surface was getting smoother upon increasing of solution concentration to $14 \mathrm{wt} \%$. With the polymer fraction lower or higher than the critical point concentration, 
bead-on-string structure or interconnected closed porous structures is occurred in the metastable region under nucleation and growth processes. At low polymer concentration ( $9-10 \mathrm{wt} \%$ ), phase separation occurred mainly through spinodal decomposition and formed a homogeneous interconnected structure with open pores.

For the initial concentration of electrospinning solution higher than $9 \mathrm{wt} \%$, an apparent bimodal pore-size distribution was observed as uniform nanopores caused by spinodal decomposition and micropores generated by subsequent coarsening process. However, the phase separation did not occur at polymer concentration higher than $13 \mathrm{wt} \%$, and favors the formation of well-defined smooth fibers. And the polymer chain mobility was inversely proportional to the polymer concentration, thus, the occurrence of bead-on-string microstructure was suppressed.

Based on the key parameters (applied voltage, flow rate and polymer concentration) introduced in the electrospinning process, this study described the optimization of smooth randomly fibers without beads or other defects. The surface "bead-on-string" structures were associated with low weight percentage of PHB in the solution, high flow rate and low applied voltage. However, the optimized condition for smooth well-defined electrospun fiber membrane as indicated from SEM micrograph in Figure 2(a) was achieved under the process parameters of $1 \mathrm{~mL} \cdot \mathrm{hr}^{-1}$ flow rate, $14 \mathrm{wt} \%$ PHB concentration, $16 \mathrm{~cm}$ collect distance and applied voltage $12 \mathrm{kV}$.

A histogram (Figure 2(b)) of the fiber diameter distribution, determined from the SEM images was estimated. In order to promote the formation of well-defined smooth fibers with uniform diameter distribution, complete solvent evaporation must be achieved along the travel path between the nozzle tip and the ground rotating drum collector. Some alterations in process parameters such as electric field increase, solution flow rate reduction effectively shorten jet travelling but enhance jet flow instability, resulting in broader fiber diameter-distribution [24].

\section{Conclusions}

In order to study the effects of PHAs production in a pilot scale reactor using dyeing wastewater as carbon source, the C:N ratios of 20:1, 40:1, 60:1, 80:1, 100:1 and 120:1 were investigated. An optimal overall production was found with C:N ratio of 100:1 and 0.058 g PHAs from unit mass of COD consumed was collected. By using the glucose to adjust the C:N, the dosage cost at the peak production was found to be $1.180 \mathrm{~g}$ polymer per RMB.

The formation of the smooth electrospun fibers can be considered as the electrospinning jet elongation by either increasing viscoelastic stress or reducing surface tension, altered by the applied electrical field strength. The viscoelastic properties through adjustment of PHB concentration are the key parameters in the process. Increasing net charge density through controlled applied voltage 
favors formation of well-defined smooth fibers. Decreasing the solution flow rate favors the formation of smooth continuous fibers without defects.

\section{Acknowledgements}

The work described in this study partially was supported by a grant from the Research Grants Council of the Hong Kong Special Administrative Region, China (UGC/FDS25/E16/17).

\section{Conflicts of Interest}

The authors declare no conflicts of interest regarding the publication of this paper.

\section{References}

[1] Wen, Q.X., Chen, Z.Q., Tian, T. and Chen, W. (2010) Effects of Phosphorus and Nitrogen Limitation on PHA Production in Activated Sludge. J. of Environ. Sci., 22, 1602-1607. https://doi.org/10.1016/S1001-0742(09)60295-3

[2] Verlinden, R.A.J., Hill, D.J., Kenward, M.A., Williams, C.D. and Radecka, I. (2007) Bacterial Synthesis of Biodegradable Polyhydroxyalkanoates. J. of Appl. Microbiol., 102, 1437-1449. https://doi.org/10.1111/j.1365-2672.2007.03335.x

[3] Shah, A.A., Hasan, F., Hameed, A. and Ahmed, S. (2008) Biological Degradation of Plastics: A Comprehensive Review. Biotechnol. Adv., 26, 246-265. https://doi.org/10.1016/j.biotechadv.2007.12.005

[4] Wu, Q., Wang, Y. and Chen, G.Q. (2009) Medical Application of Microbial Biopolyesters Polyhydroxyalkanoates. Artificial Cells, Blood Substitutes, and Biotechnology, 37, 1-12. https://doi.org/10.1080/10731190802664429

[5] Greiner, A. and Wendorff, J.H. (2007) Electrospinning: A Fascinating Method for the Preparation of Ultrathin Fibers. Angew. Chem. Int. Ed., 46, 5670-5703.

https://doi.org/10.1002/anie.200604646

[6] Larsen, G., Spreta, R. and Ortiz, R.V. (2004) Use of Coaxial Gas Jackets to Stabilize Taylor Cones of Volatile Solutions and to Induce Particle-to-Fiber Transitions. $A d v$. Mater., 16, 166-169. https://doi.org/10.1002/adma.200306021

[7] Eda, G. and Shivkumar, S. (2007) Bead-to-Fiber Transition in Electrospun Polystyrene. J. Appl. Polym. Sci., 106, 475-487. https://doi.org/10.1002/app.25907

[8] McCann, J.T., Li, D. and Xia, Y.N. (2005) Electrospinning of Nanofibers with Core-Sheath, Hollow, or Porous Structures. J. Mater. Chem., 15, 735-738. https://doi.org/10.1039/b415094e

[9] Bognitzki, M., Czado, W., Frese, T., Schaper, A., Hellwig, M., Steinhart, M. and Greiner, A. (2001) Nanostructured Fibers via Electrospinning. Adv. Mater., 13, 70-72.

https://doi.org/10.1002/1521-4095(200101)13:1<70::AID-ADMA70>3.0.CO;2-H

[10] McCann, J.T., Marquez, M. and Xia, Y.N.J. (2006) Highly Porous Fibers by Electrospinning into a Cryogenic Liquid. J. Am. Chem. Soc., 128, 1436-1437. https://doi.org/10.1021/ja056810y

[11] Dayal, P., Liu, J., Kumar, S. and Kyu, T. (2007) Experimental and Theoretical Investigations of Porous Structure Formation in Electrospun Fibers. Macromolecules, 40, 7689-7694. https://doi.org/10.1021/ma0714181

[12] Zussman, E., Rittel, D. and Yarin, A.L. (2003) Failure Modes of Electrospun Nano- 
fibers. Appl. Phys. Lett., 82, Article No. 3958. https://doi.org/10.1063/1.1579125

[13] Thompson, C.J., Chase, G.G., Yarin, A.L. and Reneker, D.H. (2007) Effects of Parameters on Nanofiber Diameter Determined from Electrospinning Model. Polymer, 48, 6913-6922. https://doi.org/10.1016/j.polymer.2007.09.017

[14] Gañán-Calvo, A.M., Dávila, J. and Barrero, A. (1997) Current and Droplet Size in the Electrospraying of Liquids. Scaling Laws. J. Aerosol Sci., 28, 249-275. https://doi.org/10.1016/S0021-8502(96)00433-8

[15] Hohman, M.M., Shin, M., Rutledge, G. and Brenner, M.P. (2001) Electrospinning and Electrically Forced Jets. II. Applications. Phys. Fluids, 13, 2221-2236. https://doi.org/10.1063/1.1384013

[16] Beachley, V. and Wen, X. (2009) Effect of Electrospinning Parameters on the Nanofiber Diameter and Length. Mater. Sci. Eng. C, 29, 663-668. https://doi.org/10.1016/j.msec.2008.10.037

[17] Inagaki, M., Yang, Y. and Kang, F. (2012) Carbon Nanofibers Prepared via Electrospinning. Adv. Mater., 24, 2547-2566. https://doi.org/10.1002/adma.201104940

[18] Deitzel, J.M., Kleinmeyer, J., Harris, D. and Beck Tan, N.C. (2001) The Effect of Processing Variables on the Morphology of Electrospun Nanofibers and Textiles. Polymer, 42, 261-272. https://doi.org/10.1016/S0032-3861(00)00250-0

[19] Mituppatham, C., Nithitanakul, M. and Supaphol, P. (2004) Ultrafine Electrospun Polyamide-6 Fibers: Effect of Solution Conditions on Morphology and Average Fiber Diameter. Macromol. Chem. Phys., 205, 2327-2338.

https://doi.org/10.1002/macp.200400225

[20] Fong, H., Chun, I. and Reneker, D.H. (1999) Beaded Nanofibers Formed during Electrospinning. Polymer, 40, 4585-4592. https://doi.org/10.1016/S0032-3861(99)00068-3

[21] Son, W.K., Youk, J.H., Lee, T.S. and Park, W.H. (2004) The Effects of Solution Properties and Polyelectrolyte on Electrospinning of Ultrafine Poly(Ethylene Oxide) Fibers. Polymer, 45, 2959-2966. https://doi.org/10.1016/j.polymer.2004.03.006

[22] Zuo, W.W., Zhu, M.F., Yang, W., Yu, H., Chen, Y.M. and Zhang, Y. (2005) Experimental Study on Relationship between Jet Instability and Formation of Beaded Fibers during Electrospinning. Polym. Eng. Sci., 45, 704-709. https://doi.org/10.1002/pen.20304

[23] Chuangchote, S., Sagawa, T. and Yoshikawa, S. (2009) Electrospinning of Poly(Vinyl Pyrrolidone): Effects of Solvents on Electrospinnability for the Fabrication of Poly(P-Phenylene Vinylene) and $\mathrm{TiO}_{2}$ Nanofibers. J. of Appl. Polym Sci., 114, 2777-2791. https://doi.org/10.1002/app.30637

[24] Ribeiro, C., Sencadas, V., Miguel Costa, C., Gomez Ribelles, J.L. and Lanceros-Mendez, S. (2011) Tailoring the Morphology and Crystallinity of Poly(L-Lactide Acid) Electrospun Membranes. Sci. Technol. Adv. Mater., 12, Article ID: 015001. https://doi.org/10.1088/1468-6996/12/1/015001 